\title{
Remission induction in acute myeloid leukemia
}

\author{
Eytan M. Stein • Martin S. Tallman
}

Received: 7 May 2012/Accepted: 24 May 2012/Published online: 13 July 2012

(C) The Japanese Society of Hematology 2012

\begin{abstract}
Inducing a complete remission (CR) in patients with acute myeloid leukemia is a prerequisite to long-term disease control with subsequent post-remission consolidation chemotherapy or allogeneic hematopoietic stem cell transplantation. The use of 7 days of infusional cytarabine and 3 days of daunorubicin or idarubicin $(7+3)$ has become the standard of care to induce CR, based on clinical trials conducted in the 1980s. Efforts to improve on the CR rate seen with the $7+3$ regimen that translates into better overall patient survival have been disappointing. Here we review recent phase III studies of novel induction strategies that show promise in increasing the rate of CR and improving disease outcome.
\end{abstract}

Keywords Acute myeloid leukemia $\cdot$ Remission

\section{Introduction}

A generally accepted prerequisite for the cure of acute myeloid leukemia (AML) is to reduce the leukemic burden by inducing a complete remission (CR) with intensive cytotoxic chemotherapy. Once an initial CR is achieved, administration of further courses of cytotoxic chemotherapy or hematopoietic stem cell transplantation (HSCT) is used to consolidate the remission and potentially achieve long-term cure. However, many older patients are unable to tolerate the intensive chemotherapy necessary to induce a CR because of comorbid health conditions, infectious

E. M. Stein · M. S. Tallman ( $\bowtie)$

Memorial Sloan Kettering Cancer Center, Leukemia Service,

1275 York Avenue, Box 380, New York, NY 10065, USA

e-mail: tallmanm@mskcc.org complications associated with marrow aplasia, and perturbations in renal and hepatic function. Indeed, over the past 40 years the cure rate of AML has steadily increased among patients younger than age 60, while the 5-year survival of older adults has remained under $10 \%$. Aside from the toxicity of chemotherapy, the poor prognosis of older adults can also be attributed to the increased incidence of AML that is biologically resistant to chemotherapy, AML that develops from antecedent hematologic disorders such as myelodysplastic syndromes (MDS) and myeloproliferative neoplasms (MPN), and therapyrelated AML. Even for older patients with "good risk" disease, the paucity of novel agents less toxic than conventional chemotherapy that are able to induce a durable $\mathrm{CR}$ is striking.

Until recently, the standard of care for patients (older and younger than 60) who can tolerate intensive remission induction therapy has remained stagnant. Studies conducted by the Cancer and Leukemia Group B (CALGB) in the 1980s established the best (although lacking) remission induction regimen to consist of 3 days of daunorubicin $\left(45 \mathrm{mg} / \mathrm{m}^{2}\right.$ or $60 \mathrm{mg} / \mathrm{m}^{2}$ ) and concomitant administration of 7 days of cytarabine $\left(100 \mathrm{mg} / \mathrm{m}^{2}\right.$ or $\left.200 \mathrm{mg} / \mathrm{m}^{2}\right)$ [1-3]. Studies since the 1980s have attempted to improve the rate of remission induction with the hope that it will lead to the clinically meaningful endpoint of improved overall survival (OS).

Various strategies have been employed to improve remission induction in AML: intensification of chemotherapy doses, adding approved agents to the standard $7+3$ regimen, and using novel agents in combination with $7+3$. Here we review novel remission induction strategies that have recently been studied in large phase III clinical trials and either have led or may lead to a change in clinical practice. 


\section{Intensification of chemotherapy doses}

One possible way to improve remission induction in AML is to give higher doses of standard $7+3$ therapy. Indeed, in 1982 Yates and colleagues studied the relative efficacy and toxicity of administering $30 \mathrm{mg} / \mathrm{m}^{2}$ versus $45 \mathrm{mg} / \mathrm{m}^{2}$ of daunorubicin. The desire to increase doses of chemotherapy was tempered by the perception that morbidity, mortality, and toxicity would offset any potential improvement in remission induction. The Eastern Cooperative Oncology Group (ECOG) sought to formally test this hypothesis in a randomized, multi-center, phase III study (trial E1900) performed between 2002 and 2008 using high doses of daunorubicin [4]. 657 patients between the ages of 17 and 60 were randomly assigned to receive daunorubicin at the standard dose of $45 \mathrm{mg} / \mathrm{m}^{2}$ or at an increased dose of $90 \mathrm{mg} / \mathrm{m}^{2}$ on days $1-3$. This was combined with cytarabine $100 \mathrm{mg} / \mathrm{m}^{2}$ given by continuous infusion on days 1-7. Patients who failed to achieve remission after the first course of induction were allowed a second induction course with the same schedule of cytarabine and $45 \mathrm{mg} / \mathrm{m}^{2}$ of daunorubicin.

Of the original 657 patients enrolled in the trial, 582 were evaluable for a response. Of these, $57.3 \%$ of patients in the standard dose daunorubicin group and $70.6 \%$ of patients in the high dose daunorubicin group achieved a CR after one or two cycles of induction chemotherapy. This remission induction translated into an OS benefit with high dose daunorubicin; the median overall survival was 15.7 months in the standard dose group and 23.7 months in the high dose group. Cytogenetic risk group at diagnosis influenced the response to high dose daunorubicin. The largest increase in OS was seen in those patients with intermediate risk cytogenetics; median survival was 17.8 months in the standard dose group and 32.3 months in the high dose group. When good risk and intermediate risk patients were pooled, the median OS was 20.7 months in the standard dose group and 34.3 months in the high dose group (Fig. 1). Disappointingly, patients with an unfavorable cytogenetic profile did not benefit from high dose daunorubicin, reinforcing that their disease resistance will likely necessitate novel agents, rather than increased doses of standard therapies.

Toxicity (grades 3-5), including cardiac toxicity, did not differ significantly between patients who received higher and lower dose daunorubicin and consisted of the standard hematologic and non-hematologic toxicities traditionally associated with induction chemotherapy. Although more patients in the high dose group had a decreased left ventricular ejection fraction (4 patients vs. 0 patients), this difference did not reach statistical significance $(p=0.06)$.

Aside from age at diagnosis, cytogenetic subcategories (favorable, intermediate, and unfavorable in the ECOG schema) have been the major determinants of prognosis in AML. In recent years, molecular mutations such as FLT-3, NPMI, IDH- 1 and IDH-2, DNMT3A and $c-K I T$ have helped refine prognostication for those patients with normal cytogenetics, and "elevated" or "lowered" patients into better or worse risk categories. As part of a larger study to develop an integrated genetic profile of patients with AML, Patel and colleagues [5], using DNA from patients treated on the E1900 protocol, investigated whether high dose versus standard dose daunorubicin influenced survival in AML patients with various molecular profiles (e.g. DNMT3A mutations). Among patients with intermediate risk disease, patients with mutant DNMT3A, as opposed to wild type, had longer OS when treated with high dose daunorubicin (Fig. 2). Similarly, patients with MLL translocations and NPMI mutations who received high dose daunorubicin also had a survival advantage, although this did not meet statistical significance when adjusted for multiple testing.

Patients older than age 60 were excluded from the E1900 study. The Dutch HOVON group in a randomized, phase III, multi-center trial published by Löwenberg and colleagues investigated the use of high dose $\left(90 \mathrm{mg} / \mathrm{m}^{2}\right)$ versus standard dose $\left(45 \mathrm{mg} / \mathrm{m}^{2}\right)$ daunorubicin (days 1-3) in patients aged 60-83 with a primary endpoint of event free survival (EFS) [6]. Treatment differences between the HOVON and ECOG trials included the use of cytarabine $200 \mathrm{mg} / \mathrm{m}^{2}$ on days 1-7 (as opposed to $100 \mathrm{mg} / \mathrm{m}^{2}$ in E1900) and a second induction round of cytarabine at a dose of $1000 \mathrm{mg} / \mathrm{m}^{2}$, twice a day for 6 days, that was administered to all patients.

Over 6 years, 813 patients enrolled in the trial. CR rates were significantly better in the high dose group than the low dose group (64 vs. $54 \%$ ). Unfortunately, this increase in CR did not translate into an increase in EFS or OS. However, in a post hoc analysis, patients aged 60-65 had an increased rate of 2-year OS with $38 \%$ surviving in the high dose group and $23 \%$ surviving in the low dose group. Various clinically meaningful measures of toxicity (30-day mortality, death during induction, incidence of serious adverse events) were similar between the two groups. Of note, differences in cardiac toxicity between high and low dose daunorubicin were not reported.

In a separate randomized phase III trial performed in Korea, Lee and colleagues [7] designed a clinical trial similar to ECOG E1900. 402 patients between the ages of 15 and 60 were randomized to receive high dose or standard dose daunorubicin on days $1-3$ with cytarabine on days 1-7. Differences in study design included an increased dose of cytarabine $\left(200 \mathrm{mg} / \mathrm{m}^{2}\right)$ and daunorubicin given as a continuous infusion. In addition, patients who failed the first induction cycle received a second induction cycle of daunorubicin at a dose of $45 \mathrm{mg} / \mathrm{m}^{2}$ for 


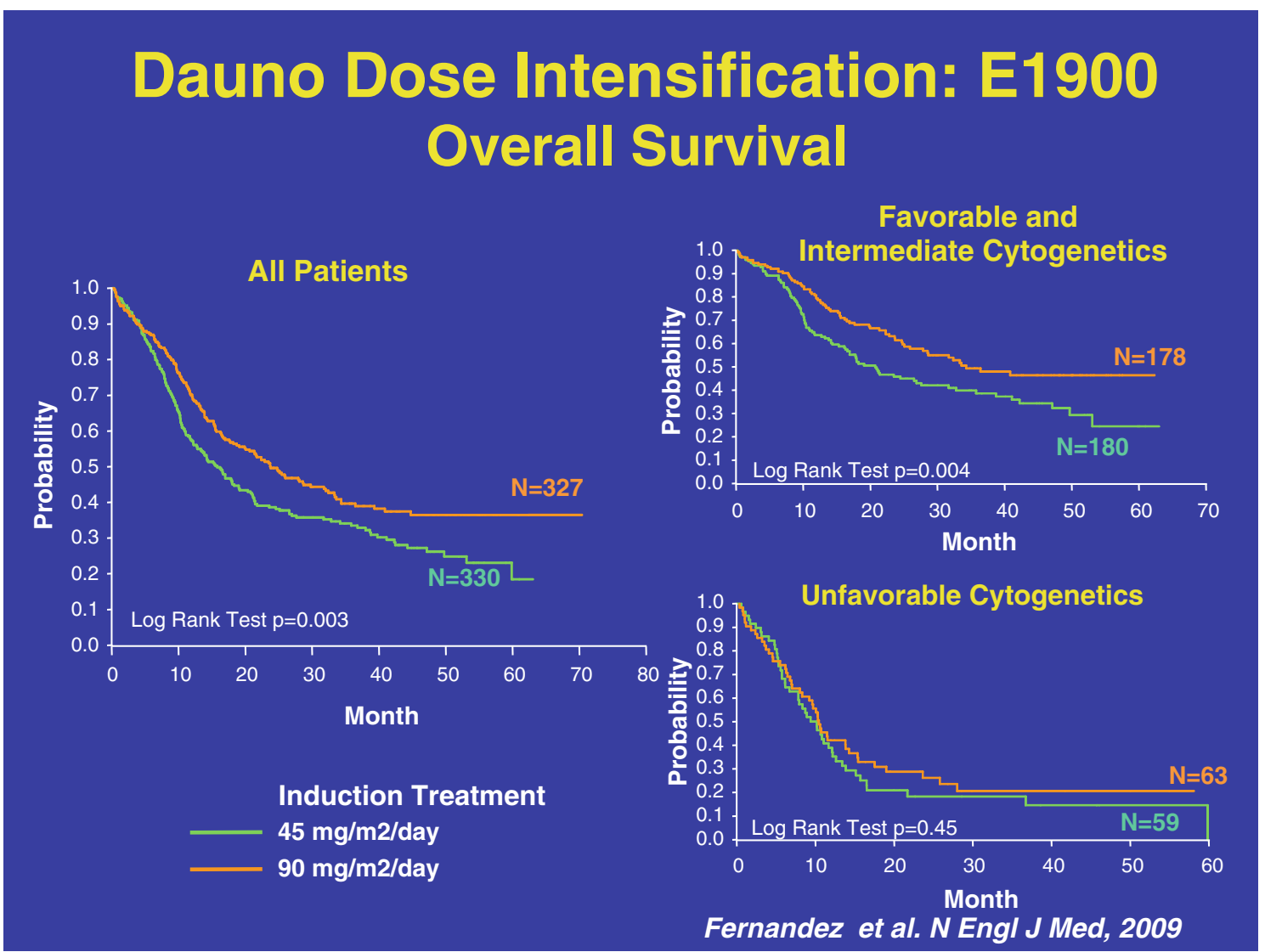

Fig. 1 Overall survival with dose intensified daunorubicin in ECOG protocol E1900

2 days and cytarabine $200 \mathrm{mg} / \mathrm{m}^{2}$ for 5 days. $82.5 \%$ of patients in the high dose group entered CR compared to $72 \%$ of patients in the standard dose group. This CR rate translated into an OS benefit in the high dose group with an estimated 5-year survival of $46.8 \%$ in the high dose group, compared to $34.6 \%$ in the standard dose group. Median OS was 35.2 months in the high dose group and 21.9 months in the low dose group. The change in OS between high dose and standard dose daunorubicin was driven by better OS in the group with intermediate risk cytogenetics. As in the ECOG trial, patients with unfavorable cytogenetics derived no benefit from higher dose therapy and somewhat surprisingly, those with favorable risk disease also did not derive a benefit from high dose therapy. The increased OS in this study (in both arms) compared to the ECOG trial may be attributable to a postconsolidation maintenance phase of treatment, although this would need to be further explored in a randomized controlled trial. There were no differences in toxicity between treatment arms.

A randomized phase III study undertaken by the Japanese Acute Leukemia Study Group (JALSG) comparing high dose daunorubicin to standard dose idarubicin was also reported in 2011 [8]. In this study, 1064 patients aged
15-64 were randomized to high dose daunorubicin $(50 \mathrm{mg} /$ $\mathrm{m}^{2}$ for 5 days) or idarubicin $\left(12 \mathrm{mg} / \mathrm{m}^{2}\right)$ on days $1-3$. Both arms received cytarabine $200 \mathrm{mg} / \mathrm{m}^{2}$ by continuous infusion on days 1-7. Thus, the cumulative doses of induction daunorubicin $\left(250 \mathrm{mg} / \mathrm{m}^{2}\right)$ were similar to the Korean and ECOG studies $\left(270 \mathrm{mg} / \mathrm{m}^{2}\right)$, but the individual, daily, treatment doses at $50 \mathrm{mg} / \mathrm{m}^{2}$ were lower. The study was powered to demonstrate non-inferiority, not superiority, of the daunorubicin arm.

1057 patients were evaluable for response. The study achieved its primary endpoint of non-inferiority with $78.2 \%$ of patients in the daunorubicin group and $77.5 \%$ of patients in the idarubicin group achieving a CR. This translated into a predicted 5-year OS (evaluated at a median follow-up of 48 months) of $48 \%$ in both the daunorubicin and idarubicin arms. Clinically meaningful toxicity favored the daunorubicin arm with more episodes of sepsis and death within 60 days in those patients who received idarubicin.

\section{Adding approved agents to standard therapy}

The Polish Acute Leukemia Group has attempted to improve the outcomes of AML by adding purine analogs to 

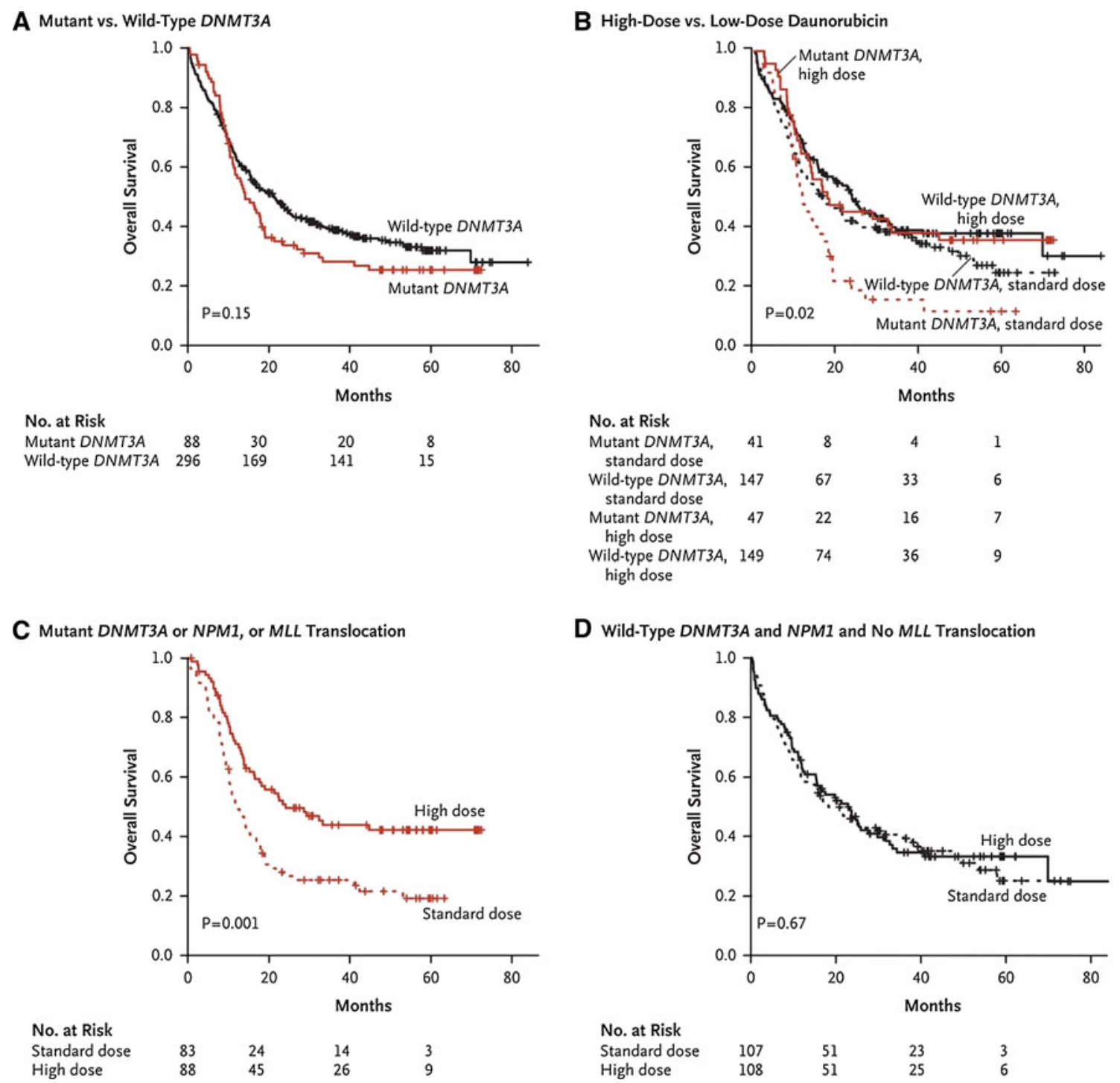

Fig. 2 Molecular determinants of response to high-dose daunorubicin induction chemotherapy

traditional induction with daunorubicin and cytarabine. In a multi-institutional, randomized, phase III study conducted in Poland between 2004 and 2008, 652 patients between the ages of 15 and 60 were randomized to daunorubicin $\left(60 \mathrm{mg} / \mathrm{m}^{2}\right.$ days $\left.1-3\right)$ and cytarabine $\left(200 \mathrm{mg} / \mathrm{m}^{2}\right.$ days $1-7$, DA group), daunorubicin and cytarabine with cladribine $\left(5 \mathrm{mg} / \mathrm{m}^{2}\right.$ days $1-5$, DAC group) or daunorubicin and cytarabine with fludarabine $\left(25 \mathrm{mg} / \mathrm{m}^{2}\right.$ days $1-5$, DAF group) [9]. The primary outcome was OS of patients comparing regimens containing purine analogs during induction to DA alone. The study was not intended to compare DAC to DAF. Patients who achieved a partial remission after one cycle of induction were allowed a second cycle of induction with the same regimen as used for induction number one.
The CR rate after DAC induction was $67.5 \%$ compared to $56 \%$ in the DA group. There was no significant difference in CR between the DA and DAF groups. The 3 -year probability of OS was $45 \%$ in the DAC arm, with a median survival of 24 months and $33 \%$ in the DA arm, with a median survival of 14 months. There was no significant difference in OS between the DAF arm and the DA arm. Toxicity was similar in the DA, DAC and DAF arms.

One limitation of this trial is that the median age of the study group was 47 years, significantly lower than the median age of patients with AML seen in population-based studies. This may limit its generalizability to the majority of patients with AML who have a median age of 68 years. It is unclear if this older patient population can tolerate purine analog therapy as well as the younger population 
seen in this study without an increase in treatment-related mortality. In addition, it is possible that doses of daunorubicin higher than $60 \mathrm{mg} / \mathrm{m}^{2}$ produce similar outcomes to adding cladribine, with decreased toxicity. This will need to be studied in future prospective randomized trials.

\section{Adding novel agents to standard therapy}

The search for novel targeted agents for AML, like tyrosine kinase inhibitors in chronic myeloid leukemia and small molecule epidermal growth factor inhibitors (such as gefitinib and erlotinib) in lung cancer, has been ongoing for many years with little success. Hope was raised in the year 2000 when gemtuzumab ozogamicin (GO), a monoclonal antibody against CD33 (which is expressed on leukemia myeloblasts) linked to calicheamicin, was approved by the United States Food and Drug Administration (FDA) under an accelerated approval for patients older than age 60 with CD33-positive AML in first relapse, and not considered candidates for cytotoxic chemotherapy. After approval, the United Kingdom Medical Research Council (UK MRC) performed a randomized trial incorporating GO $\left(3 \mathrm{mg} / \mathrm{m}^{2}\right)$ in induction and consolidation chemotherapy in a phase III, randomized, non-blinded trial of newly diagnosed patients with AML less than age 60 [10]. Although there was no difference in OS between groups, a predefined cytogenetic analysis showed a statistically significant survival benefit for patients with poor risk disease and no increased toxicity in patients who received GO. This result starkly contrasted with SWOG trial S0106, a randomized phase III trial of GO that was stopped early because of significantly increased toxicity in the GO arm with no mortality benefit. Because of this increased toxicity, Pfizer voluntarily withdrew GO from the US market.

Castaigne and colleagues have attempted to revive the benefits of GO by conducting a randomized, multi-center, phase III trial of GO in newly diagnosed patients aged 50-70 with AML, administered at $3 \mathrm{mg} / \mathrm{m}^{2}$ on days 1,4 and 7 of induction as well as on day 1 of each of two consolidation cycles with daunorubicin $60 \mathrm{mg} / \mathrm{m}^{2}$ on days 1-3 and cytarabine $200 \mathrm{mg} / \mathrm{m}^{2}$ on days 1-7 [11]. The control arm received daunorubicin and cytarabine without GO. The primary endpoint was EFS and a secondary endpoint was OS. Of note, patients with an antecedent hematologic disorder (MDS or MPN) were excluded from the trial.

280 patients were randomly assigned to each treatment group. Although there was no statistically significant difference in a combination of $\mathrm{CR}$ and $\mathrm{CR}$ with incomplete platelet recovery (CRp), the EFS and OS were significantly prolonged in the GO group. 2-year EFS was $53.2 \%$ in the GO group and $17.1 \%$ in the control group while OS at
2 years was $53.2 \%$ in the GO group and $41.9 \%$ in the control group. Prolonged neutropenia and thrombocytopenia were more common in the GO group than the control group. Liver toxicity was higher in the GO group, but did not reach statistical significance $(p=0.10)$.

The study by Castaigne has several limitations that may make it applicable to only a narrow group of AML patients. First, the age range of patients studied, between 50 and 70 years, was relatively narrow. Second, although patients had cytogenetics and immunophenotyping performed, they were eligible for the trial based on a morphological diagnosis of AML alone. Third, patients with antecedent hematologic disorders were excluded from study. Finally, the overall survival rate of patients in the control arm is strikingly higher than patients of the same age in previous clinical trials. This raises the question of whether there was bias towards enrolling only patients with the most favorable prognoses to participate in the trial. This is supported by the disparity between the EFS of $17.1 \%$ and OS of $41.9 \%$ in the control arm that suggests that those patients who had an "event" had AML with favorable biology that responded to salvage treatment. In addition, it is unclear if a higher dose of daunorubicin, as used in the ECOG and Korean phase III clinical trials, achieves the same effect as the addition of GO. Further studies should be undertaken to confirm these findings and extend its applicability to a more broad range of AML patients.

\section{Conclusion}

What can we conclude from these recent trials (Table 1)? There are now data from two large phase III trials performed in the United States and Korea that increasing the dosage of daunorubicin to $90 \mathrm{mg} / \mathrm{m}^{2}$ during induction results in increased rates of $\mathrm{CR}$ and $\mathrm{OS}$. When both trials are taken together this survival advantage is most pronounced in patients with favorable and intermediate risk disease. Determining which patients with cytogenetically normal AML with specific molecular genetic abnormalities benefit from high doses of daunorubicin during induction has been explored by Patel and colleagues but whether patients should be offered differing doses of daunorubicin based on molecular genetics alone should likely wait for confirmatory studies.

While the results of the study by Castaigne and colleagues are provocative, it is premature to advocate for a change in induction guidelines for inclusion of $\mathrm{GO}-$ not least because GO is commercially unavailable in both the United States and Europe. While there was a significant increase in OS, the results may not be generalizable to many patients with AML. In addition, the trend towards increased liver toxicity in the GO group is concerning with 


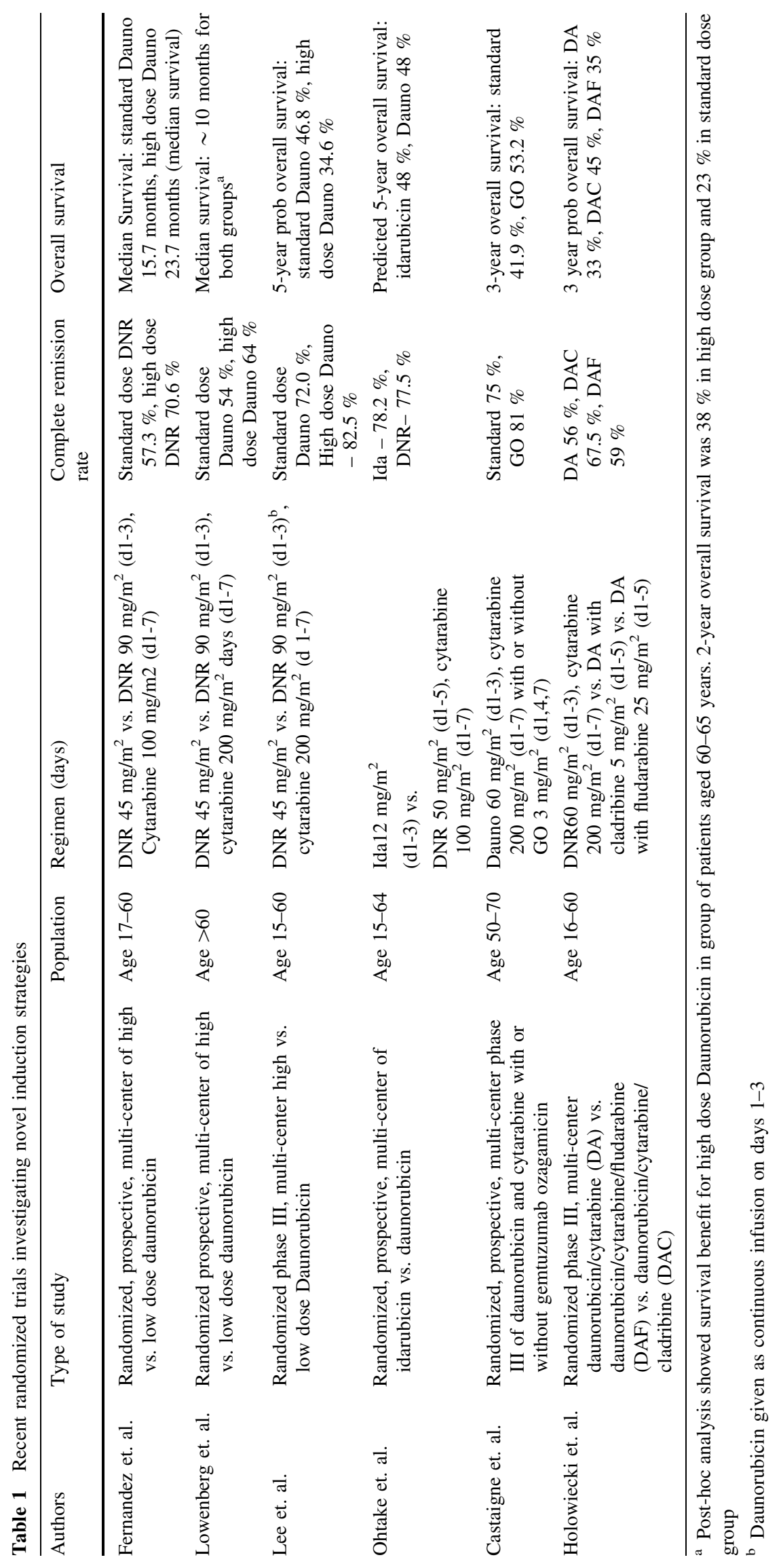


2 deaths among the 3 patents who developed veno-occlusive disease.

Because there has been no randomized trial comparing DAC or daunorubicin/cytarabine/GO with high dose daunorubicin and cytarabine, it is difficult to say which induction approach is better. It is possible that high dose daunorubicin may lead to similar outcomes as patients given GO or cladribine. Randomized clinical trials are necessary to elucidate the superior induction regimen.

\section{References}

1. Preisler $\mathrm{H}$, et al. Comparison of three remission induction regimens and two postinduction strategies for the treatment of acute nonlymphocytic leukemia: a cancer and leukemia group B study. Blood. 1987;69(5):1441-9.

2. Yates J, et al. Cytosine arabinoside with daunorubicin or adriamycin for therapy of acute myelocytic leukemia: a CALGB study. Blood. 1982;60(2):454-62.

3. Rai KR, et al. Treatment of acute myelocytic leukemia: a study by cancer and leukemia group B. Blood. 1981;58(6):1203-12.
4. Fernandez HF, et al. Anthracycline dose intensification in acute myeloid leukemia. N Engl J Med. 2009;361(13):1249-59.

5. Patel JP, et al. Prognostic relevance of integrated genetic profiling in acute myeloid leukemia. N Engl J Med. 2012;366(12): 1079-89.

6. Lowenberg B, et al. High-dose daunorubicin in older patients with acute myeloid leukemia. N Engl J Med. 2009;361(13): $1235-48$.

7. Lee $\mathrm{JH}$, et al. A randomized trial comparing standard versus highdose daunorubicin induction in patients with acute myeloid leukemia. Blood. 2011;118(14):3832-41.

8. Ohtake S, et al. Randomized study of induction therapy comparing standard-dose idarubicin with high-dose daunorubicin in adult patients with previously untreated acute myeloid leukemia: the JALSG AML201 Study. Blood. 2011;117(8):2358-65.

9. Holowiecki J, et al. Cladribine, but not fludarabine, added to daunorubicin and cytarabine during induction prolongs survival of patients with acute myeloid leukemia: a multicenter, randomized phase III study. J Clin Oncol 2012.

10. Burnett AK, et al. Identification of patients with acute myeloblastic leukemia who benefit from the addition of gemtuzumab ozogamicin: results of the MRC AML15 trial. J Clin Oncol. 2011;29(4):369-77.

11. Castaigne $\mathrm{S}$, et al. Effect of gemtuzumab ozogamicin on survival of adult patients with de novo acute myeloid leukaemia (ALFA0701): a randomised, open-label, phase 3 study. Lancet. 2012;379 (9825):1508-16. 